\title{
Borel sets with $\sigma$-compact sections for nonseparable spaces
}

\author{
by
}

\author{
Petr Holický (Praha)
}

\begin{abstract}
We prove that every (extended) Borel subset $E$ of $X \times Y$, where $X$ is complete metric and $Y$ is Polish, can be covered by countably many extended Borel sets with compact sections if the sections $E_{x}=\{y \in Y:(x, y) \in E\}, x \in X$, are $\sigma$-compact. This is a nonseparable version of a theorem of Saint Raymond. As a by-product, we get a proof of Saint Raymond's result which does not use transfinite induction.
\end{abstract}

1. Introduction. We prove a nonseparable version of the theorem $[5$, Theorem] of Saint Raymond. Saint Raymond's theorem states in particular that each Borel subset $B$ of the product of Polish spaces $X$ and $Y$ with $\sigma$-compact sections $B_{x}=\{y \in Y:(x, y) \in B\}, x \in X$, can be covered by countably many Borel sets with compact sections. It says even more, namely that an analytic set $A \subset X \times Y$ can be separated from another analytic set $B \subset X \times Y$ by a countable union of Borel sets with compact sections if the sections of $A$ can be separated from the sections of $B$ by $\sigma$-compact sets.

Our result generalizes that of Saint Raymond to the case of nonseparable complete metric spaces $X$ and extended Borel sets $E \subset X \times Y$. Our proof does not use the analogue of the Cantor derivatives used in Saint Raymond's proof and thus it is different even in the separable case. The reason for our modification is to avoid a cardinality argument which cannot be used for the nonseparable case. In fact, several attempts to get the uniformization presented here, or stronger results corresponding to known improvements of Saint Raymond's theorem in the classical setting, failed because of the lack of a suitable substitute of the boundedness principle for coanalytic ranks. Therefore we proceed similarly to [3, Theorem 3.1], where we proved a nonseparable version of a theorem of Luzin and Novikov on (extended)

2000 Mathematics Subject Classification: Primary 54H05; Secondary 54C65, 28A05.

Key words and phrases: extended Borel sets, $\sigma$-compact sections, nonseparable metric spaces.

The work is a part of the research project MSM 0021620839 financed by MSMT and it was also partly supported by GAČR 201/06/0198 and GAČR 201/06/0018. 
Borel sets with countable sections. We work however also with Suslin sets instead of just (extended) Borel ones. Adjusting our proof from [3] in a similar fashion, we get a nonseparable version of Luzin-Novikov's theorem for Suslin sets.

2. Suslin and extended Borel sets in metric spaces. A subset $S$ of a topological space $X$ is a Suslin set if

$$
S=\bigcup_{\left(n_{1}, n_{2}, \ldots\right) \in \mathbb{N}^{\mathbb{N}}} \bigcap_{k \in \mathbb{N}} F_{n_{1}, \ldots, n_{k}},
$$

where $F_{n_{1}, \ldots, n_{k}}$ are closed subsets of $X$. It is well known that $S \subset X$ is Suslin if and only if it is the projection of a closed subset of $X \times P$, where $P$ is a separable completely metrizable (i.e., Polish) space.

The set $S$ is co-Suslin in $X$ if its complement is Suslin in $X$. If $S$ and its complement in $X$ are Suslin, we say that $S$ is a bi-Suslin set in $X$. Let us recall that Suslin subsets of Polish spaces are called analytic spaces and that bi-Suslin sets in an analytic space coincide with its Borel subsets.

The class of extended Borel subsets of a metric space is the smallest class containing Borel sets which is a $\sigma$-field and which is closed under the unions of discrete families (see, e.g., [2]).

We recall a lemma ([3, Lemma 2.1]) on projections along separable metric spaces which we need later. Let us introduce some notation first.

Considering spaces $X$ and $Y$, we use $\pi_{X}$ and $\pi_{Y}$ to denote the projections of $X \times Y$ to $X$ and $Y$, respectively.

In what follows we say that a family $\mathcal{F}$ of subsets of a metric space $X$ is discrete (more precisely metrically discrete) if there is an $\varepsilon>0$ such that the distance of any two elements of $\mathcal{F}$ is greater than or equal to $\varepsilon$. An indexed family $\left(F_{a}: a \in A\right)$ is discrete if there is an $\varepsilon>0$ such that the distance of $F_{a}$ and $F_{b}$ is greater than or equal to $\varepsilon$ for every pair $a, b$ of distinct elements of $A$ (the distance is infinite if one of the sets is empty). We then say that the family $\mathcal{F}$, or the indexed family $\left(F_{a}: a \in A\right)$, is $\varepsilon$-discrete.

The above-mentioned lemma now reads as follows.

Lemma 2.1. Let $X$ be a metric space and $Y$ be a separable metric space. If the indexed family $\left(D_{a}: a \in A\right)$ of subsets of $X \times Y$ is discrete, then there are sets $D_{a}(n) \subset X \times Y$ such that

(a) $D_{a}=\bigcup_{n \in \mathbb{N}} D_{a}(n)$,

(b) $D_{a}(n)=D_{a} \cap(P \times U)$ for some open sets $P$ in $X$ and $U$ in $Y$,

(c) $\left(\pi_{X}\left(D_{a}(n)\right): a \in A\right)$ is discrete for every $n \in \mathbb{N}$.

The description of $D_{a}(n)$ 's in (b) ensures that they are relatively open in $D_{a}$, and thus open, extended Borel, or Suslin, if $D_{a}$ 's were of the respective type. 
We still need another simple fact about discrete families in metric spaces. The rather artificial formulation with an auxiliary set $Z$ suits well our applications.

Lemma 2.2. Let $X$ be a metric space and $Z$ an arbitrary set. Let $\mathcal{S}=$ $\bigcup\left\{\mathcal{S}_{n}: n \in \mathbb{N}\right\}$ be a family of subsets of $X \times Z$ such that the indexed family $\left(\pi_{X}(S): S \in \mathcal{S}_{n}\right)$ is $\varepsilon_{n}$-discrete in $X$ for every $n \in \mathbb{N}$. Set

$$
P_{\mathcal{F}}=\bigcap\left\{\overline{\pi_{X}(S)}: S \in \mathcal{F}\right\}
$$

for every finite $\mathcal{F} \subset \mathcal{S}$. Then the indexed family

$$
\left(P_{\mathcal{F}}: \mathcal{F} \text { a finite subfamily of } \mathcal{S}\right)
$$

is $\sigma$-discrete.

Proof. Given a finite family $\mathcal{F} \subset \mathcal{S}$, the set $P_{\mathcal{F}}$ may be nonempty only if each $\mathcal{S}_{n}$ contains at most one element of $\mathcal{F}$. Fix distinct $k_{1}, \ldots, k_{n} \in \mathbb{N}$. It is not difficult to check that the indexed family $\left(P_{\left\{F_{1}, \ldots, F_{n}\right\}}: F_{i} \in \mathcal{S}_{k_{i}}\right.$, $i=1, \ldots, n)$ is $\varepsilon$-discrete, where $\varepsilon=\min \left\{\varepsilon_{k_{i}}: i=1, \ldots, n\right\}$.

We describe a particular representation of Suslin subsets of the product of a complete metric space with a Polish space together with some notation.

Lemma 2.3. Let $S$ be a Suslin subset of $X \times Y$, where $(X, \varrho)$ is a complete metric space and $(Y, \sigma)$ is a complete separable metric space. Then there is a closed subset $F(S)$ of $(X \times Y) \times \mathbb{N}^{\mathbb{N}}$ and an open base $\mathcal{C}(S)$ of $F(S)$ such that the indexed family $\left(\pi_{X}(C): C \in \mathcal{C}(S)\right)$ is $\sigma$-discrete. (We then denote by $\pi_{S}$ the restriction of $\pi_{X \times Y}$ to $F$.) Consider the metric $\max (\varrho, \sigma, \tau)$ on $X \times Y \times \mathbb{N}^{\mathbb{N}}$, where $\tau$ is a complete metric on $\mathbb{N}^{\mathbb{N}}$ compatible with the product topology. If $C_{n} \in \mathcal{C}(S)$ are nonempty such that $\lim _{n \rightarrow \infty} \operatorname{diam} C_{n}=0$ and $\bar{C}_{n+1} \subset C_{n}$ for $n \in \mathbb{N}$, then $\bigcap_{n \in \mathbb{N}} \pi_{S}\left(C_{n}\right)$ is a singleton in $S$.

Proof. There is a closed set $F(S) \subset(X \times Y) \times \mathbb{N}^{\mathbb{N}}$ such that $S=$ $\pi_{X \times Y}(F(S))$. As $F(S)$ is a metric space, it has a $\sigma$-discrete open base $\mathcal{B}$. Since $Y \times \mathbb{N}^{\mathbb{N}}$ is separable, we may find, by Lemma 2.1, an open refinement $\mathcal{C}(S)$ of $\mathcal{B}$, and thus also an open base of $F(S)$, such that $\left(\pi_{X}(C): C \in \mathcal{C}(S)\right)$ is $\sigma$-discrete. The remaining statement follows by the completeness of $F(S)$ and continuity of $\pi_{S}$.

We need a separation theorem of Hansell, a nonseparable version of the reduction theorem for co-Suslin sets, and a nonseparable version of the classical Novikov approximation theorem (cf. [5, Lemma 2]).

THEOREM 2.4 .

(a) $E$ is extended Borel in a complete metric space $X$ if and only if $E$ is bi-Suslin in $X$. 
(b) If $C_{n}, n \in \mathbb{N}$, are co-Suslin subsets of a metric space $X$, then there are pairwise disjoint co-Suslin sets $D_{n} \subset C_{n}$ such that $\bigcup_{n \in \mathbb{N}} D_{n}=$ $\bigcup_{n \in \mathbb{N}} C_{n}$.

(c) Let $S_{n}$ be Suslin sets in a metric space $X$ with $\bigcap\left\{S_{n}: n \in \mathbb{N}\right\}=\emptyset$. Then there are bi-Suslin sets $E_{n}$ in $X$ such that $S_{n} \subset E_{n}$ and $\bigcap\left\{E_{n}\right.$ : $n \in \mathbb{N}\}=\emptyset$.

Proof. Statement (a) follows from [1, Corollary 4.11 and Theorem 4.15]. Statement (b) follows easily from the nonseparable uniformization theorem (see, e.g., [4, Theorem 7]) applied to the set $\bigcup\left\{C_{n} \times\{n\}: n \in \mathbb{N}\right\}$.

The last statement follows from (b) applied to the sets $C_{n}=X \backslash S_{n}$. The sets $D_{n}$ from (b) form a partition of $X$ consisting of bi-Suslin sets. The complements $E_{n}$ of $D_{n}$ are the desired sets.

3. A nonseparable version of the Saint Raymond theorem. Our main result is the following improvement of the Saint Raymond theorem. We say that $A \subset Z$ is separated from $B \subset Z$ by a set $C \subset Z$, or that $C$ separates $A$ from $B$, if $A \subset C \subset Z \backslash B$.

TheOREM 3.1. Let $S$ and $T$ be Suslin subsets of the product of a complete metric space $X$ and a Polish space $Y$ such that $S_{x}$ can be separated from $T_{x}$ by a $\sigma$-compact set for every $x \in X$. Then there are extended Borel sets $E_{n}, n \in \mathbb{N}$, such that $\left(E_{n}\right)_{x}$ is compact for each $x \in X$ and $E=\bigcup_{n \in \mathbb{N}} E_{n}$ separates $S$ from $T$.

The following corollary is of particular interest.

TheOREM 3.2. Let $E \subset X \times Y$ be an extended Borel subset of the product of a complete metric space $X$ and a Polish space $Y$ such that the sections $E_{x}, x \in X$, are $\sigma$-compact. Then there are extended Borel sets $E_{n}, n \in \mathbb{N}$, such that each $\left(E_{n}\right)_{x}$ is compact and $\bigcup_{n \in \mathbb{N}} E_{n}=E$.

Proof. We apply the preceding theorem to $S=E$ and $T=(X \times Y) \backslash E$. The set $E$ is necessarily the separating set from Theorem 3.1 and so it is a countable union of extended Borel sets with compact sections.

We may suppose that $Y$ is compact and metric. Indeed, let $\widehat{Y}$ be a metric compactification of $Y$. Put $\widehat{T}=T \cup(X \times(\widehat{Y} \backslash Y))$. Note that $S$ and $\widehat{T}$ are Suslin in $X \times \widehat{Y}$ since $Y$ is a $G_{\delta}$ subset of $\widehat{Y}$. Separating $S$ and $\widehat{T}$ as in Theorem 3.1, we get extended Borel sets $E_{n}$ in $X \times \widehat{Y}$, even in $X \times Y$, with compact sections.

Further, for $T \subset X \times Y$, we denote by $\mathcal{E}_{T}$ the family of all extended Borel subsets $E$ of $X \times Y$ with compact sections $E_{x}, x \in X$, which do not intersect $T$. The conclusion of Theorem 3.1 may be restated by saying that there is an $E \in\left(\mathcal{E}_{T}\right)_{\sigma}$ with $S \subset E$. We show that $\left(\mathcal{E}_{T}\right)_{\sigma}$ coincides with a formally larger family now. 
LEMMA 3.3. Let $X$ be a metric space, $Y$ a separable metric space and $T \subset X \times Y$. Then $\left(\mathcal{E}_{T}\right)_{\sigma}=\left\{E: E=\bigcup \mathcal{D}, \mathcal{D} \subset \mathcal{E}_{T}\right.$ is $\sigma$-discrete $\}$.

Proof. The inclusion $\left(\mathcal{E}_{T}\right)_{\sigma} \subset\left\{E: E=\bigcup \mathcal{D}, \mathcal{D} \subset \mathcal{E}_{T}\right.$ is $\sigma$-discrete $\}$ is obvious. To prove the other one, let $\mathcal{D}$ be a $\sigma$-discrete family of elements of $\mathcal{E}_{T}$. Let $\bigcup_{n \in \mathbb{N}} \mathcal{E}_{n}$ be a refinement of $\mathcal{D}$ such that $\left(\pi_{X}(E): E \in \mathcal{E}_{n}\right)$ is discrete as in Lemma 2.1. Every element $E \in \mathcal{E}_{n}$ is a subset of an element $D(E) \in \mathcal{D}$. Thus the set $\bar{E} \cap D(E)$ is in $\mathcal{E}_{T}$ and the union $E_{n}=\bigcup\{\bar{E} \cap D(E)$ : $\left.E \in \mathcal{E}_{n}\right\}$ is in $\mathcal{E}_{T}$ as well. Now $\bigcup \mathcal{D}=\bigcup_{n \in \mathbb{N}} E_{n} \in\left(\mathcal{E}_{T}\right)_{\sigma}$ and the lemma is proved.

Similarly to $[5$, Lemma 1$]$, we deduce that the vertical closures $\bar{A}^{v}=$ $\bigcup\left\{\{x\} \times \bar{A}_{x}: x \in X\right\}$ of Suslin sets are Suslin sets.

Lemma 3.4. Let $X$ be metric, $Y$ Polish, and $A \subset X \times Y$ be Suslin. Then the set $\bar{A}^{v}$ is Suslin.

Proof. Let $\mathcal{U}_{n}$ be a countable open cover of $Y$ consisting of sets with diameter less than $1 / n$. The sets $A \cap(X \times U)$ are Suslin for $U \in \mathcal{U}_{n}$, and so their projections $A(U)$ to $X$ are Suslin. Now

$$
\bar{A}^{v}=\bigcap_{n \in \mathbb{N}} \bigcup_{U \in \mathcal{U}_{n}} A(U) \times \bar{U}
$$

is Suslin.

As in [5, Lemma 3], we first need to prove a separation theorem for sets with compact sections which follows from the (nonseparable) Novikov and separation theorems. We can repeat the proof of Saint Raymond almost word for word by simply referring to nonseparable variants of the needed theorems. Let us remark that if we had the Saint Raymond lemma [5, Lemma 3] proved for a separable metric space $X$, with (extended) Borel replaced by bi-Suslin, we could derive the nonseparable version using a separable factorization described in [4, Corollary 2].

THEOREM 3.5. Let $X$ be metric, $Y$ compact, and $S, T \subset X \times Y$ be Suslin such that $\bar{S}_{x} \cap T_{x}=\emptyset$ for every $x \in X$. Then there is a bi-Suslin set $E \subset X \times Y$ with compact sections $E_{x}, x \in X$, which separates $S$ from $T$.

Proof. Let $\left\{U_{i}^{n}: i=1, \ldots, i_{n}\right\}$ be an open cover of $Y$ by open sets of diameter less than $1 / n$ for $n \in \mathbb{N}$. Define $S_{i}^{n}=\pi_{X}\left(S \cap\left(X \times \overline{U_{i}^{n}}\right)\right) \times \overline{U_{i}^{n}}$ and $S_{n}=\bigcup\left\{S_{i}^{n}: i=1, \ldots, i_{n}\right\}$. Since the closure of $S_{x}$ does not intersect $T_{x}$ for every $x \in X$, it is easy to check that $\bar{S}^{v}=\bigcap\left\{S_{n}: n \in \mathbb{N}\right\}$ as in the proof of Lemma 3.4, and $\bigcap\left\{S_{n} \cap T: n \in \mathbb{N}\right\}=\emptyset$. Due to Theorem 2.4(c), we find bi-Suslin sets $N_{n} \supset S_{n} \cap T$ such that $\bigcap\left\{N_{n}: n \in \mathbb{N}\right\}=\emptyset$. Using the notation $T_{i}^{n}$ for the set $\left(T \cap\left(X \times \overline{U_{i}^{n}}\right)\right) \backslash N_{n}$, we get by Theorem 2.4(c) a bi-Suslin set $B_{i}^{n}$ separating $\pi_{X}\left(S_{i}^{n}\right)$ from $\pi_{X}\left(T_{i}^{n}\right)$. We may now check easily 
that the set $E=\bigcap_{n \in \mathbb{N}} \bigcup\left\{B_{i}^{n} \times \overline{U_{i}^{n}}: i=1, \ldots, i_{n}\right\}$ fulfils the requirements of our theorem.

REMARK 3.6. Theorem 3.5 and its proof can be used to prove that the projection of an extended Borel set with compact sections in the product $X \times Y$ of a complete metric space $X$ and a Polish space $Y$ is extended Borel (cf. the remark following [5, Lemma 3]). Using now our Theorem 3.2, we get the nonseparable version of the theorem of Arsenin-Kunugui saying that the projection to $X$ of an extended Borel subset of $X \times Y$ with $\sigma$ compact sections is extended Borel (cf. [4, Theorem 6], where another proof was given). We may now improve Theorem 3.2 so that the sets $E_{n}$ have the same projection as $E$ in the notation of the theorem. Indeed, we may extend each $E_{n}$ obtained in the theorem to

$$
E_{n} \cup \bigcup\left\{E_{m} \backslash\left(\pi_{X}\left(\bigcup\left\{E_{k} \cup E_{n}: k<m\right\}\right) \times Y\right): m \in \mathbb{N}\right\} .
$$

These new sets might be called "compact-valued uniformizations of $E$ ". Also a nonseparable version of Shchegol'kov's uniformization theorem for such sets $E$ could be proved similarly to [5, Corollary 12].

Let us point out that by the preceding considerations (see Lemmas 3.3 and 3.4) to prove Theorem 3.1 it is sufficient to find a $\sigma$-discrete family $\mathcal{S}$ of Suslin sets such that $\bigcup\left\{\bar{S}^{v}: S \in \mathcal{S}\right\}$ separates $S$ from $T$.

Let $X$ be a complete metric space, $Y$ a compact metric space, and $S, T$ be Suslin subsets of $X \times Y$ as in the assumptions of Theorem 3.1. Assuming that $S$ cannot be separated from $T$ by an element of $\left(\mathcal{E}_{T}\right)_{\sigma}$ (as in Theorem 3.1), we are going to construct a homeomorphic copy $H$ of the Cantor set in $Y$ such that $H \subset S_{x} \cup T_{x}$ and $H \cap T_{x}$ is a countable dense subset of $H$ for an $x \in X$. This shows that $S_{x}$ is not $\sigma$-compact, which is a contradiction (as otherwise the nonempty set $H \cap T_{x}$ would be $G_{\delta}$ in $H$ and of first category in itself at the same time). To construct $H$, we shall look for a "Hurewicz-like scheme" (cf. [5, Lemma 8]).

We apply the above representation Lemma 2.3 for the Suslin sets $S$ and $T$, and we fix a choice of the closed subsets $F(S)$ and $F(T)$ of $(X \times Y) \times \mathbb{N}^{\mathbb{N}}$, and of the open bases $\mathcal{C}(S)$ of $F(S)$ and $\mathcal{C}(T)$ of $F(T)$ as in Lemma 2.3. The restrictions of $\pi_{X \times Y}$ to $F(S)$ and $F(T)$ are denoted by $\pi_{S}$ and $\pi_{T}$, respectively. We consider the corresponding maximum metrics in products of metric spaces as in Lemma 2.3.

Let $U(A, r)$ denote the open $r$-neighbourhood of $A$ for $r>0$. We use $\mathbf{i}^{\wedge} j$ to denote the sequence $\left(i_{1}, \ldots, i_{n}, j\right)$ whenever $\mathbf{i}=\left(i_{1}, \ldots, i_{n}\right) \in\{0,1\}^{n}$ for some $n \in \mathbb{N}$ and $j \in\{0,1\}$.

Definition 3.7. An indexed family

$$
\left(\left(S_{\mathbf{j}}, T_{\mathbf{j}}, C_{\mathbf{j}}, D_{\mathbf{j}}\right): \mathbf{j} \in\{0,1\}^{n}, n \in \mathbb{N}\right)
$$


of quadruples of sets such that $S_{\mathbf{j}}, T_{\mathbf{j}} \subset X \times Y, C_{\mathbf{j}} \in \mathcal{C}(S)$, and $D_{\mathbf{j}} \in \mathcal{C}(T)$ forms a Hurewicz-like scheme if for every $n \in \mathbb{N}$, using the abbreviation $\mathbf{i}$ for $\left(i_{1}, \ldots, i_{n-1}\right) \in\{0,1\}^{n-1}$, we have

(a) $S_{\mathbf{i}^{\wedge} 1}=\pi_{S}\left(C_{\mathbf{i}^{\wedge} 1}\right) \subset S_{\mathbf{i}}, C_{\emptyset}=F(S), C_{i_{1}, \ldots, i_{k}, 0}=C_{i_{1}, \ldots, i_{k}}$ for $k<n$, $\bar{C}_{\mathbf{i}^{\wedge} 1} \subset C_{\mathbf{i}}$, and $\operatorname{diam} C_{\mathbf{i}^{\wedge} 1}<1 / n$. In particular, diam $S_{\mathbf{i}^{\wedge} 1}<1 / n$.

(b) $T_{\mathbf{i} \wedge 0}=\pi_{T}\left(D_{\mathbf{i}^{\wedge} 0}\right)\left(\subset T_{\mathbf{i}}\right), D_{\emptyset}=F(T), D_{i_{1}, \ldots, i_{k}, 1}=F(T)$ for $k<n$, $\bar{D}_{\mathbf{i}^{\wedge} 0} \subset D_{\mathbf{i}}$, and diam $D_{\mathbf{i}^{\wedge} 0}<1 / n$. In particular, diam $T_{\mathbf{i}^{\wedge} 0}<1 / n$ and, if $i_{n-1}=0, T_{\mathbf{i}^{\wedge} 0} \subset T_{\mathbf{i}}$.

(c) $S_{\mathbf{i}^{\wedge} 0}=S_{\mathbf{i}} \cap U\left(T_{\mathbf{i}^{\wedge} 0}, r_{\mathbf{i}^{\wedge} 0}\right)$, where $r_{\mathbf{i} \wedge}=\max \left\{1 / k: k \in \mathbb{N}, \operatorname{diam} U\left(T_{\mathbf{i}^{\wedge} 0}, 1 / k\right)<1 / n\right.$, $\left.1 / k<(1 / 3) \operatorname{dist}\left(\pi_{Y}\left(S_{\mathbf{i}^{\wedge} 1}\right), \pi_{Y}\left(T_{\mathbf{i}^{\wedge} 0}\right)\right)\right\}$.

In particular, $\operatorname{diam} S_{\mathbf{i}^{\wedge} 0}<1 / n$ and $S_{\mathbf{i}^{\wedge} 0} \subset S_{\mathbf{i}}$.

(d) $T_{\mathbf{i} \wedge 1}=T \cap U\left(S_{\mathbf{i} \wedge 1}, r_{\mathbf{i} \wedge 1}\right)$, where $r_{\mathbf{i}^{\wedge} 1}=\max \left\{1 / k: k \in \mathbb{N}, \operatorname{diam} U\left(S_{\mathbf{i}^{\wedge} 1}, 1 / k\right)<1 / n\right.$, $\left.1 / k<(1 / 3) \operatorname{dist}\left(\pi_{Y}\left(S_{\mathbf{i}^{\wedge} 1}\right), \pi_{Y}\left(T_{\mathbf{i}^{\wedge} 0}\right)\right)\right\}$.

In particular, $\operatorname{diam} T_{\mathbf{i}^{\wedge} 1}<1 / n$ and $T_{\mathbf{i}^{\wedge} 1} \subset T$.

(e) The family

$$
\left(\overline{\pi_{Y}\left(S_{\mathbf{j}} \cup T_{\mathbf{j}}\right)}: \mathbf{j} \in\{0,1\}^{n}\right)
$$

is disjoint.

(f)

$$
\bigcap\left\{\pi_{X}\left({\overline{S_{\mathbf{j}}}}^{v} \cap T_{\mathbf{j}}\right): \mathbf{j} \in\{0,1\}^{n}\right\} \neq \emptyset
$$

Let us remark that we could weaken some requirements of Definition 3.7. In particular, item (d) could be omitted for our purposes. Nevertheless, we use (d) to make some of the formulations (for example that of (e)) simpler. Similarly, we could define $D_{i_{1}, \ldots, i_{k}}$ if $i_{k}=0$ only. In fact, the Hurewicz-like scheme is uniquely determined by the sets $C_{\mathbf{i}^{\wedge} 1}$ and $D_{\mathbf{i}^{\wedge} 0}$ for $\mathbf{i} \in\{0,1\}^{k}$, $k=0,1, \ldots$.

We say that an indexed family

$$
\mathcal{P}_{n}=\left(\left(S_{\mathbf{i}}, T_{\mathbf{i}}, C_{\mathbf{i}}, D_{\mathbf{i}}\right): \mathbf{i} \in\{0,1\}^{k}, k \leq n\right)
$$

is admissible if it meets the requirements (a) to (e) of the above Definition 3.7 restricted to $\mathcal{P}_{n}$.

The family

$$
\mathcal{Q}=\left(\left(S_{\mathbf{k}}, T_{\mathbf{k}}, C_{\mathbf{k}}, D_{\mathbf{k}}\right): \mathbf{k} \in\{0,1\}^{n+1}\right)
$$

is an admissible extension of the admissible family $\mathcal{P}_{n}$ from (1) if the family

$$
\mathcal{P}_{n+1}=\left(\left(S_{\mathbf{i}}, T_{\mathbf{i}}, C_{\mathbf{i}}, D_{\mathbf{i}}\right): \mathbf{i} \in\{0,1\}^{k}, k \leq n+1\right)
$$

is admissible.

Similarly to [5, Lemma 7], we prove 
Lemma 3.8. Let the indexed family

$$
\left(\left(S_{\mathbf{j}}, T_{\mathbf{j}}, C_{\mathbf{j}}, D_{\mathbf{j}}\right): \mathbf{j} \in\{0,1\}^{n}, n \in \mathbb{N}\right)
$$

form a Hurewicz-like scheme. Then there is a homeomorphic copy $H$ of the Cantor set such that $H \subset S_{x} \cup T_{x}$ and $H \cap T_{x}$ is a countable dense subset of $H$ for some $x \in X$.

Proof. We are going to define a mapping $\varphi:\{0,1\}^{\mathbb{N}} \rightarrow X \times Y$ first. Let $\left(i_{1}, i_{2}, \ldots\right) \in\{0,1\}^{\mathbb{N}}$.

If $\left(i_{1}, i_{2}, \ldots\right) \in I_{0}$, where $I_{0}=\left\{\left(i_{1}, i_{2}, \ldots\right) \in\{0,1\}^{\mathbb{N}}\right.$ : there is an $n_{0} \in \mathbb{N}$ such that $i_{n}=0$ if $\left.n \geq n_{0}\right\}$, we set $\varphi\left(i_{1}, i_{2}, \ldots\right)$ equal to the unique element of $\bigcap\left\{\bar{T}_{i_{1}, \ldots, i_{n}}: n \geq n_{0}\right\} \subset T$ (cf. Lemma 2.3). Here $n_{0}$ stands, e.g., for the smallest $n_{0}$ from the definition of $I_{0}$. This definition is correct because the sets $T_{i_{1}, \ldots, i_{n}}, n \geq n_{0}$, form a decreasing sequence of sets with diameters tending to zero by (b) and they are nonempty by (f) of Definition 3.7.

If $\left(i_{1}, i_{2}, \ldots\right) \in\{0,1\}^{\mathbb{N}} \backslash I_{0}$, i.e., the sequence contains infinitely many one's, then we define $\varphi\left(i_{1}, i_{2}, \ldots\right)$ as the unique element of $\bigcap\left\{\bar{S}_{i_{1}, \ldots, i_{n}}\right.$ : $n \in \mathbb{N}\} \subset S$ (cf. Lemma 2.3). This is correct because the sets $S_{i_{1}, \ldots, i_{n}}$, $n \in \mathbb{N}$, form a decreasing sequence of sets with diameters tending to zero by (a) and (c), and they are nonempty by (f) of Definition 3.7.

We now prove that $\varphi$ is a homeomorphism of $\{0,1\}^{\mathbb{N}}$ onto a subset of $S_{x} \cup T_{x}$ for some $x \in X$. It follows from (e) of Definition 3.7 that $\varphi$ is injective.

As each

$$
S_{i_{1}, \ldots, i_{n}} \cup T_{i_{1}, \ldots, i_{n}} \subset U\left(S_{i_{1}, \ldots, i_{n}}, 1 / n\right)
$$

by (f), (b), and (d) of Definition 3.7, and diam $S_{i_{1}, \ldots, i_{n}}<1 / n$ by (a) and (c), we may easily check that $\varphi$ is continuous.

We see from the definition of $\varphi$ that

$$
\pi_{X}\left(\varphi\left(\{0,1\}^{\mathbb{N}}\right)\right) \subset \bigcup\left\{\pi_{X}\left(\bar{S}_{\mathbf{j}}\right) \cup \pi_{X}\left(\overline{T_{\mathbf{j}}}\right): \mathbf{j} \in\{0,1\}^{n}\right\}
$$

for every $n \in \mathbb{N}$. Given a fixed $n \in \mathbb{N}$, the family of all sets $\pi_{X}\left(\bar{S}_{\mathbf{j}}\right)$ and $\pi_{X}\left(\overline{T_{\mathbf{j}}}\right)$ for $\mathbf{j} \in\{0,1\}^{n}$ has nonempty intersection by (f). All these sets have diameter less than $1 / n$ by (a)-(d). Thus the projection to $X$ of $\varphi\left(\{0,1\}^{\mathbb{N}}\right)$ has diameter less than $2 / n$ for every $n \in \mathbb{N}$, and so it is a singleton.

Now, $H=\pi_{Y}\left(\varphi\left(\{0,1\}^{\mathbb{N}}\right)\right)$ is the required set. Indeed, $H \cap T_{x}=\pi_{Y} \circ \varphi\left(I_{0}\right)$ is countable and dense in $H$ since $I_{0}$ is countable and dense in $\{0,1\}^{\mathbb{N}}$, and $\pi_{Y} \circ \varphi\left(\{0,1\}^{\mathbb{N}} \backslash I_{0}\right)=H \cap S_{x}$.

An important role in our approach is played by the following notions related to property (f) of Definition 3.7. 


\section{DEFINITION 3.9.}

(a) An indexed family $\mathcal{P}=\left(\left(S_{i}, T_{i}\right): i \in\{1, \ldots, n\}\right)$ of pairs of subsets of $X \times Y$ is partially separated if

$$
(\forall x \in X)(\exists i \in\{1, \ldots, n\}) \overline{\left(S_{i}\right)_{x}} \cap\left(T_{i}\right)_{x}=\emptyset .
$$

(b) We say that an indexed family $\left(\left(S_{i}, T_{i}\right): i \in\{1, \ldots, n\}\right)$ of pairs of subsets of $X \times Y$ can be partially separated if there is a set $C \in$ $\left(\mathcal{E}_{T}\right)_{\sigma}$ such that the family $\left(\left(S_{i} \backslash C, T_{i}\right): i \in\{1, \ldots, n\}\right)$ is partially separated.

Note that this definition does not depend on whether we consider the family as an indexed family or just as the set of pairs of sets $S_{i}$ and $T_{i}$. We prefer to speak about indexed families because we are going to apply these notions to subfamilies of the Hurewicz-like scheme which is an indexed family.

We prove a lemma concerning partial separation of families of the particular form $\left(\left(S_{i}, T\right): i \in\{1, \ldots, n\}\right)$.

Lemma 3.10. Let $S_{i} \subset X \times Y, i \in\{1, \ldots, n\}$, be Suslin sets such that

$$
(\forall x \in X)(\exists i \in\{1, \ldots, n\}) \overline{\left(S_{i}\right)_{x}} \cap T_{x}=\emptyset .
$$

Then there is a set $D \in \mathcal{E}_{T}$ such that

$$
(\forall x \in X)(\exists i \in\{1, \ldots, n\})\left(S_{i} \backslash D\right)_{x}=\emptyset .
$$

Proof. We consider the sets $C_{i}=\left\{x \in X: \overline{\left(S_{i}\right)_{x}} \cap T_{x}=\emptyset\right\}$. The complement of $C_{i}$ is Suslin in $X$ since it is the projection of the intersection of the Suslin sets $T$ and $\bar{S}_{i}^{v}$ (cf. Lemma 3.4).

The sets $C_{i}, i=1, \ldots, n$, cover $X$ by our assumptions. By the reduction theorem (Theorem 2.4(b) above) there are complements of Suslin sets $B_{i} \subset C_{i}$ which form a partition of $X$. The separation theorem recalled in Theorem 2.4(a) shows that all $B_{i}$ 's are extended Borel.

Applying Theorem 3.5 to the pairs of Suslin sets $S_{i}^{\prime}=S_{i} \cap\left(B_{i} \times Y\right)$ and $T$, we get sets $E_{i} \in \mathcal{E}_{T}$ with $S_{i}^{\prime} \subset E_{i}$. The set $D=\bigcup\left\{E_{i}: i=1, \ldots, n\right\}$ satisfies our requirements.

We conclude our proof of Theorem 3.1 by proving the inductive step of the construction of a Hurewicz-like scheme. Recall that we assume that the conclusion of the theorem does not hold. So our construction may and shall begin with the choice $S_{\emptyset}=S, T_{\emptyset}=T, C_{\emptyset}=F(S)$, and $D_{\emptyset}=F(T)$. We say that a family

$$
\left(\left(S_{\mathbf{i}}, T_{\mathbf{i}}, C_{\mathbf{i}}, D_{\mathbf{i}}\right): \mathbf{i} \in\{0,1\}^{k}\right)
$$

of quadruples is (can be) partially separated if the family $\left(\left(S_{\mathbf{i}}, T_{\mathbf{i}}\right): \mathbf{i} \in\right.$ $\left.\{0,1\}^{k}\right)$ is (can be) partially separated, respectively. 
Lemma 3.11. Let $n \in\{0,1, \ldots\}$ and

$$
\mathcal{P}_{n}=\left(\left(S_{\mathbf{i}}, T_{\mathbf{i}}, C_{\mathbf{i}}, D_{\mathbf{i}}\right): \mathbf{i} \in\{0,1\}^{k}, k=0,1, \ldots, n\right)
$$

be an admissible family such that the family

$$
\left(\left(S_{\mathbf{j}}, T_{\mathbf{j}}\right): \mathbf{j} \in\{0,1\}^{n}\right)
$$

cannot be partially separated. Then there is an admissible extension

$$
\left(\left(S_{\mathbf{k}}, T_{\mathbf{k}}, C_{\mathbf{k}}, D_{\mathbf{k}}\right): \mathbf{k} \in\{0,1\}^{n+1}\right)
$$

of $\mathcal{P}_{n}$ which cannot be partially separated.

Proof. Suppose that every admissible extension $\mathcal{Q}$ of $\mathcal{P}_{n}$ can be partially separated. We choose for every such extension

$$
\mathcal{Q}=\left(\left(S_{\mathbf{k}}, T_{\mathbf{k}}, C_{\mathbf{k}}, D_{\mathbf{k}}\right): \mathbf{k} \in\{0,1\}^{n+1}\right)
$$

a set $C(\mathcal{Q}) \in\left(\mathcal{E}_{T}\right)_{\sigma}$ as in Definition 3.9(b), thus we have

$$
(\forall x \in X)\left(\exists \mathbf{k} \in\{0,1\}^{n+1}\right) \overline{\left(S_{\mathbf{k}} \backslash C(\mathcal{Q})\right)_{x}} \cap\left(T_{\mathbf{k}}\right)_{x}=\emptyset .
$$

We put

$$
\mathcal{F}(\mathcal{Q})=\left\{C_{\mathbf{j}^{\wedge} 1}: \mathbf{j} \in\{0,1\}^{n}\right\} \cup\left\{D_{\mathbf{j}^{\wedge} 0}: \mathbf{j} \in\{0,1\}^{n}\right\} .
$$

By Lemma 2.2 applied to the family $\mathcal{S}=\{C: C \in \mathcal{C}(S)\} \cup\{D: D \in \mathcal{C}(T)\}$ of subsets of $X \times Z$ with $Z=Y \times \mathbb{N}^{\mathbb{N}}$, the indexed family

$$
\left(P_{\mathcal{F}(\mathcal{Q})}: \mathcal{Q} \text { is an admissible extension of } \mathcal{P}_{n}\right),
$$

and thus also the indexed family $\left(P_{\mathcal{F}(\mathcal{Q})} \times Y: \mathcal{Q}\right.$ is an admissible extension of $\left.\mathcal{P}_{n}\right)$, is $\sigma$-discrete. Note that $\mathcal{Q}$ is uniquely given by a choice of $\left(\left(C_{\mathbf{j}^{\wedge} 1}, D_{\mathbf{j}^{\wedge} 0}\right)\right.$ : $\mathbf{j} \in\{0,1\}^{n}$ ) due to Definition 3.7.

Replacing $C(\mathcal{Q})$ by its intersection with the closed set $P_{\mathcal{F}(\mathcal{Q})} \times Y$, we may and shall assume that

$$
C(\mathcal{Q}) \subset P_{\mathcal{F}(\mathcal{Q})} \times Y
$$

Thus the family $\left(C(\mathcal{Q}): \mathcal{Q}\right.$ an admissible extension of $\left.\mathcal{P}_{n}\right)$ becomes $\sigma$ discrete and, by Lemma 3.3, the set $C=\bigcup\{C(\mathcal{Q}): \mathcal{Q}$ an admissible extension of $\left.\mathcal{P}_{n}\right\}$ belongs to $\left(\mathcal{E}_{T}\right)_{\sigma}$.

We define

$$
N=\left\{x \in X:\left(\forall \mathbf{j} \in\{0,1\}^{n}\right) \overline{\left(S_{\mathbf{j}} \backslash C\right)_{x}} \cap\left(T_{\mathbf{j}}\right)_{x} \neq \emptyset\right\} .
$$

Note that $N$ is Suslin in $X$ as a finite intersection of projections to $X$ of the Suslin sets ${\overline{\left(S_{\mathbf{j}} \backslash C\right)}}^{v} \cap T_{\mathbf{j}}$ (cf. Lemma 3.4).

Consider families

$$
\mathcal{R}=\left(\left(C_{\mathbf{j}^{\wedge} 1}^{0}, C_{\mathbf{j}^{\wedge} 1}^{1}\right) \in \mathcal{C}(S) \times \mathcal{C}(S): \mathbf{j} \in\{0,1\}^{n}\right)
$$

of pairs of sets such that, for $\mathbf{j} \in\{0,1\}^{n}$ and $j=0,1$,

• $S_{\mathbf{j}^{\wedge} 1}^{j}=\pi_{S}\left(C_{\mathbf{j}^{\wedge} 1}^{j}\right) \subset S_{\mathbf{j}}$, 
- $\overline{C_{\mathbf{j}^{\wedge} 1}^{j}} \subset C_{\mathbf{j}}$,

- $\operatorname{diam} C_{\mathbf{j}^{\wedge} 1}^{j}<1 /(n+1)$

(as in (a) of the definition of the Hurewicz-like scheme; the sets $S_{\mathbf{j}^{\wedge} 1}^{j}$ are "potential candidates for $S_{\mathbf{j} \wedge 1}$ ") such that moreover

$$
\overline{\pi_{Y}\left(S_{\mathbf{j}^{\wedge} 1}^{0}\right)} \cap \overline{\pi_{Y}\left(S_{\mathbf{j}^{\wedge} 1}^{1}\right)}=\emptyset .
$$

We denote by $\mathfrak{R}$ the set of all such families $\mathcal{R}$.

Suppose that an

$$
\mathcal{R}=\left(\left(C_{\mathbf{j}^{\wedge} 1}^{0}, C_{\mathbf{j}^{\wedge} 1}^{1}\right): \mathbf{j} \in\{0,1\}^{n}\right) \in \mathfrak{R}
$$

is fixed and $S_{\mathbf{j}^{\wedge} 1}^{j}=\pi_{S}\left(C_{\mathbf{j}^{\wedge} 1}^{j}\right)$. In the case that

$$
(\forall x \in N)\left(\exists \mathbf{j} \in\{0,1\}^{n}\right)(\exists j=0,1) \overline{\left(S_{\mathbf{j}^{\wedge} 1}^{j} \backslash C\right)_{x}} \cap T_{x}=\emptyset,
$$

we know from Lemma 3.10 applied to the family of Suslin sets

$$
\left(S_{\mathbf{j}^{\wedge} 1}^{j} \backslash C\right) \cap(N \times Y)
$$

that there is a set $D(\mathcal{R}) \in \mathcal{E}_{T}$ such that

$$
(\forall x \in N)\left(\exists \mathbf{j} \in\{0,1\}^{n}\right)(\exists j=0,1)\left(S_{\mathbf{j}^{\wedge} 1}^{j} \backslash(C \cup D(\mathcal{R}))\right)_{x}=\emptyset .
$$

We may and do assume that $\mathcal{D}(\mathcal{R}) \subset P_{\mathcal{F}(\mathcal{R})} \times Y$ by replacing it with its intersection with $P_{\mathcal{F}(\mathcal{R})} \times Y$, where $\mathcal{F}(\mathcal{R})=\left\{C_{\mathbf{j}^{\wedge} 1}^{j}: j=0,1, \mathbf{j} \in\{0,1\}^{n}\right\}$ and $P_{\mathcal{F}(\mathcal{R})}$ is the closed set defined in Lemma 2.2 (we use here $\{C: C \in \mathcal{C}(S)\}$ as the family $\mathcal{S}$ of subsets of $X \times Z$, with $Z=Y \times \mathbb{N}^{\mathbb{N}}$, in that lemma). This ensures that the family $(D(\mathcal{R}): \mathcal{R} \in \mathfrak{R})$ is $\sigma$-discrete, and so its union $D=\bigcup\{D(\mathcal{R}): \mathcal{R} \in \mathfrak{R}\}$ is in $\left(\mathcal{E}_{T}\right)_{\sigma}$ due to Lemma 3.3.

Since the family

$$
\left(\left(S_{\mathbf{j}}, T_{\mathbf{j}}\right): \mathbf{j} \in\{0,1\}^{n}\right)
$$

cannot be partially separated by our assumptions, also the family

$$
\left(\left(S_{\mathbf{j}} \backslash(C \cup D), T_{\mathbf{j}}\right): \mathbf{j} \in\{0,1\}^{n}\right)
$$

is not partially separated. Thus there is an $x \in X$ such that

$$
\left(\forall \mathbf{j} \in\{0,1\}^{n}\right) \overline{\left(S_{\mathbf{j}} \backslash(C \cup D)\right)_{x}} \cap\left(T_{\mathbf{j}}\right)_{x} \neq \emptyset .
$$

Necessarily, $x \in N$. Consequently, there are distinct

$$
s_{\mathbf{j}^{\wedge} 1}^{j} \in\left(S_{\mathbf{j}} \backslash(C \cup D)\right)_{x}, \quad j=0,1,
$$

for every $\mathbf{j} \in\{0,1\}^{n}$. We choose a family

$$
\mathcal{R}_{0}=\left(\left(C_{\mathbf{j}^{\wedge} 1}^{0}, C_{\mathbf{j}^{\wedge} 1}^{1}\right): \mathbf{j} \in\{0,1\}^{n}\right) \in \mathfrak{R}
$$


such that $S_{\mathbf{j}^{\wedge} 1}^{j}=\pi_{S}\left(C_{\mathbf{j}^{\wedge} 1}^{j}\right) \ni s_{\mathbf{j}^{\wedge} 1}^{j}$. This is possible since $S_{\mathbf{j}}$ is a relatively open subset of $\pi_{S}\left(C_{\mathbf{j}}\right)$ and $\mathcal{C}(S)$ is an open base of $F(S)$ (Lemma 2.3). We have

$$
\left(\forall \mathbf{j} \in\{0,1\}^{n}\right)(\forall j=0,1)\left(S_{\mathbf{j}^{\wedge} 1}^{j} \backslash(C \cup D)\right)_{x} \neq \emptyset .
$$

This implies the negation of (4) as $D\left(\mathcal{R}_{0}\right) \subset D$ and our choice of $\mathcal{R}_{0}$ ensures the negation of (3), i.e., that

$$
\left(\exists x_{0} \in N\right)\left(\forall \mathbf{j} \in\{0,1\}^{n}\right)(\forall j=0,1) \overline{\left(S_{\mathbf{j}^{\wedge} 1}^{j} \backslash C\right)_{x_{0}}} \cap T_{x_{0}} \neq \emptyset .
$$

As $x_{0} \in N$, we also have a

$$
t_{\mathbf{j}^{\wedge} 0} \in \overline{\left(S_{\mathbf{j}} \backslash C\right)_{x_{0}}} \cap\left(T_{\mathbf{j}}\right)_{x_{0}}
$$

for every $\mathbf{j} \in\{0,1\}^{n}$. We may now choose $S_{\mathbf{j}^{\wedge} 1}$ equal either to $S_{\mathbf{j}^{\wedge} 1}^{0}$ or to $S_{\mathbf{j}^{\wedge} 1}^{1}$ so that $t_{\mathbf{j} \wedge 0} \notin \overline{\left(S_{\mathbf{j}^{\wedge} 1}\right)_{x_{0}}}$ for each $\mathbf{j}=\left(i_{1}, \ldots, i_{n}\right) \in\{0,1\}^{n}$.

We choose $D_{\mathbf{j} \wedge 0} \in \mathcal{C}(T)$ so that

- $T_{\mathbf{j} \wedge} 0=\pi_{T}\left(D_{\mathbf{j}^{\wedge} 0}\right) \ni\left(x_{0}, t_{\mathbf{j}^{\wedge} 0}\right)$,

- $\bar{D}_{\mathbf{j} \wedge 0} \subset D_{\mathbf{j}}$,

- $\operatorname{diam} D_{\mathbf{j} \wedge}<1 /(n+1)$

(i.e., satisfying assumptions (b) from the definition of the Hurewicz-like scheme). This is possible since $T_{\mathbf{j}}=\pi_{T}\left(D_{\mathbf{j}}\right)$ if $i_{n}=0$ (and $T_{\mathbf{j}}$ is a relatively open subset of $T$ if $i_{n}=1$ ), and $\mathcal{C}(T)$ is an open base of $F(T)$ from Lemma 2.3.

For $\mathbf{j} \in\{0,1\}^{n}$, let $S_{\mathbf{j} \wedge 0}$ and $T_{\mathbf{j} \wedge 1}$ be the sets uniquely prescribed by (c) and (d) of Definition 3.7, and $C_{\mathbf{j}^{\wedge} 0}=C_{\mathbf{j}}, D_{\mathbf{j}^{\wedge} 1}=F(T)$ as in (a) and (b) of Definition 3.7.

Conditions (a)-(f) are now satisfied and we denote the admissible extension $\left(\left(S_{\mathbf{k}}, T_{\mathbf{k}}, C_{\mathbf{k}}, D_{\mathbf{k}}\right): \mathbf{k} \in\{0,1\}^{n+1}\right)$ by $\mathcal{Q}_{0}$.

Moreover, the family $\left(\left(S_{\mathbf{k}} \backslash C, T_{\mathbf{k}}\right): \mathbf{k} \in\{0,1\}^{n+1}\right)$ is not partially separated since, for every $\mathbf{j} \in\{0,1\}^{n}$,

$$
\overline{\left(S_{\mathbf{j} \wedge 0} \backslash C\right)_{x_{0}}} \cap\left(T_{\mathbf{j}^{\wedge} 0}\right)_{x_{0}} \ni t_{\mathbf{j} \wedge 0}
$$

by (6) as $S_{\mathbf{j} \wedge 0}$ is an open neighbourhood of $T_{\mathbf{j} \wedge 0} \ni\left(x_{0}, t_{\mathbf{j} \wedge 0}\right)$ intersected with $S_{\mathbf{j}}$, and

$$
\overline{\left(S_{\mathbf{j}^{\wedge} 1} \backslash C\right)_{x_{0}}} \cap T_{x_{0}} \neq \emptyset
$$

by (5).

This contradicts $(2)$ since $C\left(\mathcal{Q}_{0}\right) \subset C$, and the lemma is proved.

4. Luzin-Novikov theorem for Suslin sets. Using some ideas of the proof of Theorem 3.1, we improve [3, Theorem 3.1] (Luzin-Novikov theorem for extended Borel sets) to get the following nonseparable version of the Luzin-Novikov theorem for Suslin sets. 
TheOREM 4.1. Let $S$ be a Suslin subset of the product of a complete metric space $X$ and a Polish space $Y$ such that $S_{x}$ is a countable set for every $x \in X$. Then there are Suslin sets $A_{n}, n \in \mathbb{N}$, such that each $\left(A_{n}\right)_{x}$ contains at most one point for every $x \in X$ and $S=\bigcup_{n \in \mathbb{N}} A_{n}$.

As a corollary we get a "nonseparable separation version" of the LuzinNovikov theorem.

Corollary 4.2. Let $S$ and $T$ be two disjoint Suslin subsets of the product of a complete metric space $X$ and a Polish space $Y$ such that $S_{x}$ is a countable set for every $x \in X$. Then there are extended Borel sets $E_{n}, n \in \mathbb{N}$, such that each $\left(E_{n}\right)_{x}, x \in X$, contains at most one point, $S \subset \bigcup_{n \in \mathbb{N}} E_{n}$, and $\bigcup_{n \in \mathbb{N}} E_{n} \cap T=\emptyset$.

It is clear that Theorem 4.1 is an immediate consequence of Corollary 4.2 since we may choose $T=\emptyset$, use Corollary 4.2 to get $E_{n}$ 's, and put $A_{n}=S \cap E_{n}$. We shall however prove Theorem 4.1 first and observe that using Lemma 4.3 below, we get Corollary 4.2 as an easy consequence of Theorem 4.1 (we may put $E_{n}=\widehat{A}_{n}$ for $A_{n}$ 's from Theorem 4.1). Lemma 4.3 says in particular that a Suslin graph $S$ can be extended to an extended Borel one. This is a well known result in the classical case because $S$ is necessarily the graph of a Borel measurable mapping from the analytic projection $\pi_{X}(S)$ into $Y$ if $X$ is Polish, and it is well known that it can be extended to a Borel measurable mapping of the whole $X$ into $Y$. The statement of the following lemma follows by combining several known results. However, we give a direct proof for the convenience of the reader.

Lemma 4.3. Let $S, T \subset X \times Y$ be disjoint Suslin subsets of the product of a metric space $X$ and a Polish space $Y$, and suppose the sections $S_{x}$, $x \in X$, contain at most one point each. Then there is a bi-Suslin set $\widehat{S}$ such that $S \subset \widehat{S},(\widehat{S})_{x}$ contains at most one point for every $x \in X$, and $\widehat{S} \cap T=\emptyset$.

Proof. Fix a sequence of countable partitions $\mathcal{U}_{n}, n \in \mathbb{N}$, of $Y$ into Suslin sets (or even into differences of open sets) such that each $\mathcal{U}_{n}$ consists of sets with diameter less than $1 / n$.

The Suslin sets $S_{U}=\pi_{X}(S \cap(X \times U)), U \in \mathcal{U}_{n}$, form a countable partition of $\pi_{X}(S)$ for every $n \in \mathbb{N}$. Using the separation theorem for pairs of Suslin sets (see, e.g., Theorem 2.4(c) for pairs of disjoint sets) inductively, we find bi-Suslin sets $B_{U} \supset S_{U}, U \in \mathcal{U}_{n}$, in $X$ which are pairwise disjoint. The set

$$
G=\bigcap_{n \in \mathbb{N}} \bigcup\left\{B_{U} \times U: U \in \mathcal{U}_{n}\right\}
$$

is bi-Suslin, $S \subset G$, and $G_{x}$ contains at most one point for every $x \in X$. 
By the separation theorem (see, e.g., Theorem 2.4(c) again), there is a bi-Suslin set $H \subset X \times Y$ such that $S \subset H$ and $H \cap T=\emptyset$. The set $E=G \cap H$ fulfils all requirements of our lemma.

The next lemma plays a similar role in this section as Lemma 3.10 in the previous one.

Lemma 4.4. Let $S_{1}, \ldots, S_{n}$ be Suslin subsets of $X \times Y$, where $X$ is a metric space, $Y$ a Polish space, and let

$$
(\forall x \in X)(\exists i \in\{1, \ldots, n\}) \text { card }\left(S_{i}\right)_{x} \leq 1 .
$$

Then there is a bi-Suslin set $E \subset X \times Y$ such that

- $E_{x}$ contains at most one point for every $x \in X$;

- $(\forall x \in X)(\exists i \in\{1, \ldots, n\}) S_{i} \backslash E=\emptyset$.

Proof. Let $\mathcal{B}$ be a countable open base of $Y$. The sets $\left\{x \in X:\left(S_{i}\right)_{x} \cap\right.$ $U_{j} \neq \emptyset$ if $\left.j=0,1\right\}, i=1, \ldots, n$, are Suslin for all pairs $U_{0}, U_{1}$ of disjoint (nonempty) elements of $\mathcal{B}$, and so their union is the Suslin set $\{x \in X$ : card $\left.\left(S_{i}\right)_{x}>1\right\}$. So the sets $C_{i}=\left\{x \in X: \operatorname{card}\left(S_{i}\right)_{x} \leq 1\right\}$ are co-Suslin and, by our assumptions, they form a cover of $X$. Theorem 2.4(b), (c) ensures the existence of a partition of $X$ consisting of bi-Suslin sets $D_{i} \subset C_{i}$. Now, let $\widehat{S}_{i} \supset S_{i}$ be a bi-Suslin set such that $\left(\widehat{S}_{i}\right)_{x}, x \in X$, contains at most one point (we use Lemma 4.3 with $S=S_{i}$ and $T=\emptyset$ ). The set $E=\bigcup_{i=1}^{n} \widehat{S}_{i} \cap\left(D_{i} \times Y\right)$ is as desired.

To prove Theorem 4.1 we proceed by contradiction. We suppose that the conclusion of the theorem is false for some $S$.

Let $F(S), \mathcal{C}(S)$, and $\pi_{S}$ form the representation of $S$ as in Lemma 2.3 above.

We are going to find a Cantor-like scheme by choosing $C_{\mathbf{j}} \in \mathcal{C}(S), \mathbf{j} \in$ $\{0,1\}^{n}$, such that, for every $n \in \mathbb{N}$ and $\mathbf{j}=\left(i_{1}, \ldots, i_{n}\right) \in\{0,1\}^{n}$,

$\left(\mathrm{a}_{n}\right) S_{\mathbf{j}}=\pi_{S}\left(C_{\mathbf{j}}\right)$

$\left(\mathrm{b}_{n}\right) \bar{C}_{i_{1}, \ldots, i_{n}} \subset C_{i_{1}, \ldots, i_{n-1}}$ and $\operatorname{diam} C_{i_{1}, \ldots, i_{n}}<1 / n$;

$\left(\mathrm{c}_{n}\right)\left(\overline{\pi_{Y}\left(S_{\mathbf{j}}\right)}: \mathbf{j} \in\{0,1\}^{n}\right)$ is disjoint;

$\left(\mathrm{d}_{n}\right) \bigcap\left\{\pi_{X}\left(S_{\mathbf{j}}\right): \mathbf{j} \in\{0,1\}^{n}\right\} \neq \emptyset$.

We say that the family $\mathcal{P}_{n}=\left(S_{\mathbf{j}}: \mathbf{j} \in\{0,1\}^{n}\right)$ is partially covered if for every $x \in X$ there is a $\mathbf{j} \in\{0,1\}^{n}$ such that $\left(S_{\mathbf{j}}\right)_{x}=\emptyset$. We say that the family can be partially covered if there are bi-Suslin graphs $G_{k}$ of mappings from $X$ to $Y$ such that $\left(S_{\mathbf{j}} \backslash \bigcup_{k \in \mathbb{N}} G_{k}: \mathbf{j} \in\{0,1\}^{n}\right)$ is partially covered. Denote by $\mathcal{E}$ the set of all bi-Suslin graphs of mappings from $X$ to $Y$.

Proceeding similarly to the proof of Theorem 3.1 above, we can find a Cantor-like scheme by induction. We begin by choosing $S_{\emptyset}=S$ and $C_{\emptyset}=$ $F(S)$. 
Lemma 4.5. Let $\mathcal{P}_{n}=\left(C_{\mathbf{i}}: k \leq n, \mathbf{i} \in\{0,1\}^{k}\right)$ for some $n=0,1, \ldots$ satisfy $\left(\mathrm{a}_{k}\right)-\left(\mathrm{d}_{k}\right)$ for $k=1, \ldots, n$ and suppose $\left(S_{\mathbf{j}}=\pi_{S}\left(C_{\mathbf{j}}\right): \mathbf{j} \in\{0,1\}^{n}\right)$ cannot be partially covered. Then there is an extension $\mathcal{Q}=\left(C_{\mathbf{k}}: \mathbf{k} \in\right.$ $\left.\{0,1\}^{n+1}\right)$ of $\mathcal{P}_{n}$ such that $\left(S_{\mathbf{k}}=\pi_{S}\left(C_{\mathbf{k}}\right): \mathbf{k} \in\{0,1\}^{n+1}\right)$ cannot be partially covered and such that $\mathcal{P}_{n+1}=\left(C_{\mathbf{i}}: k \leq n+1, \mathbf{i} \in\{0,1\}^{k}\right)$ satisfies $\left(\mathrm{a}_{k}\right)-\left(\mathrm{d}_{k}\right)$ for $k=1, \ldots, n+1$.

Proof. We proceed by contradiction. Suppose that for every extension $\mathcal{Q}$ which gives $\mathcal{P}_{n+1}$ satisfying $\left(\mathrm{a}_{k}\right)-\left(\mathrm{d}_{k}\right)$ for $k=1, \ldots, n+1$, there is a set $C(\mathcal{Q}) \in \mathcal{E}_{\sigma}$ such that $\left(\pi_{S}\left(C_{\mathbf{k}}\right) \backslash C(\mathcal{Q}): \mathbf{k} \in\{0,1\}^{n+1}\right)$ is partially covered. Let $P_{\mathcal{Q}}$ be the sets defined in Lemma 2.2 applied to $\mathcal{F}=\mathcal{Q}$ and $\mathcal{S}=\mathcal{C}(S)$. We may restrict ourselves to possibly smaller bi-Suslin $C(\mathcal{Q})$ such that $C(\mathcal{Q}) \subset$ $P_{\mathcal{Q}} \times Y$, and we get $C$ as the union of the sets $C(\mathcal{Q})$ over all admissible extensions $\mathcal{Q}$ of $\mathcal{P}_{n}$. The family $\left(\pi_{S}(C(\mathcal{Q})): \mathcal{Q}\right.$ an admissible extension of $\left.\mathcal{P}_{n}\right)$ is $\sigma$-discrete and thus $C$ belongs to $\mathcal{E}_{\sigma}$.

In the case that

$$
(\forall x \in X)\left(\exists \mathbf{j} \in\{0,1\}^{n}\right) \operatorname{card}\left(S_{\mathbf{j}} \backslash C\right)_{x}<2,
$$

we see by Lemma 4.4 that $\left(S_{\mathbf{j}}: \mathbf{j} \in\{0,1\}^{n}\right)$ can be partially covered, a contradiction.

In the case that

$$
\left(\exists x_{0} \in X\right)\left(\forall \mathbf{j} \in\{0,1\}^{n}\right) \operatorname{card}\left(S_{\mathbf{j}} \backslash C\right)_{x_{0}} \geq 2,
$$

we easily find, using the fact that $\mathcal{C}(S)$ is an open base of $F(S)$ and $\pi_{S}$ is continuous, an admissible extension $\mathcal{Q}_{0}=\left(C_{\mathbf{k}}: \mathbf{k} \in\{0,1\}^{n+1}\right)$ of $\mathcal{P}_{n}$ so that $\left(\pi_{S}\left(C_{\mathbf{j}^{\wedge} i}\right)\right)_{x_{0}} \cap\left(S_{\mathbf{j}} \backslash C\right)_{x_{0}} \neq \emptyset$ for $\mathbf{j} \in\{0,1\}^{n}$ and $i \in\{0,1\}$. Thus $\left(\pi_{S}\left(C_{k}\right) \backslash C: k \in\{0,1\}^{n+1}\right)$ is not partially covered. This contradicts our choice of $C \supset C\left(\mathcal{Q}_{0}\right)$, and the lemma is proved.

Now, having a Cantor-like scheme, the set

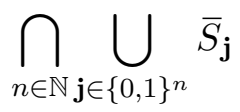

is a homeomorphic copy of the Cantor set contained in an $S_{x_{0}}$; as $S_{x_{0}}$ is countable, this is a contradiction.

The author thanks to J. Spurný and M. Zelený for many useful comments during the process of preparation of this note.

\section{References}

[1] R. W. Hansell, On the non-separable theory of $k$-Borel and $k$-Souslin sets, General Topology Appl. 3 (1973), 161-195.

[2] -, On characterizing non-separable analytic and extended Borel sets as types of continuous images, Proc. London Math. Soc. 28 (1974), 683-699. 
[3] P. Holický, Borel sets with countable sections for nonseparable spaces, Proc. Amer. Math. Soc. 134 (2006), 1519-1525.

[4] P. Holický and V. Komínek, On projections of nonseparable Souslin and Borel sets along separable spaces, Acta Univ. Carolin. Math. Phys. 42 (2001), 33-41.

[5] J. Saint Raymond, Boreliens à coupes $K_{\sigma}$, Bull. Soc. Math. France 104 (1976), 389400 .

Department of Mathematical Analysis

Faculty of Mathematics and Physics

Charles University

Sokolovská 83

18675 Praha 8, Czech Republic

E-mail: holicky@karlin.mff.cuni.cz

Received 28 July 2006 